Original Paper http://ajol.info/index.php/ijbcs http://indexmedicus.afro.who.int

\title{
Diversité, abondance et distribution des touracos (Musophagidae) dans quelques milieux naturels du centre et du sud-est de la Côte d'Ivoire
}

\author{
Okon Modeste OKON, Ekoun Michael KONAN, Kouakou Pierre KOUADIO et \\ Hilaire K. YAOKOKORE-BEIBRO*
}

UR de Biologie de la Conservation et Gestion de la Faune, Laboratoire de Zoologie et Biologie Animale, UFR Biosciences de l'Université Félix Houphouët-Boigny, Abidjan-Cocody, 22 BP 582 Abidjan 22, Côte d'Ivoire.

*Auteur correspondant; E-mail: hyaokokore@yahoo.fr, Tel : +22507634265

\section{RESUME}

L'écologie et la zoogéographie des touracos sont peu connues en Côte d'Ivoire. Ainsi, en vue de déterminer la diversité, l'abondance et la distribution des touracos, une étude a été réalisée dans les parcs nationaux du Banco et d'Azagny, dans la Réserve Scientifique de Lamto et dans la Forêt classée de YapoAbbé, d'août 2014 à juillet 2015. Dans ces différents milieux, l'inventaire des espèces de touracos appartenant à la famille des Musophagidae a été effectué à travers la méthode des indices ponctuels d'abondance (IPA). Au total, cinq espèces de touracos ont été inventoriées. Ce sont : Tauraco macrorhynchus, Tauraco persa, Corythaeola cristata, Crinifer piscator et Musophaga violacea. Les résultats indiquent que la Réserve Scientifique de Lamto a la plus grande richesse en touracos et que Tauraco macrorhynchus est la seule espèce commune à l'ensemble des sites. Par contre, Musophaga violacea est présent uniquement dans la Réserve Scientifique de Lamto. L'abondance des touracos varie d'un site à l'autre et selon les habitats. Par ailleurs, les espèces de touracos ne sont pas distribuées de la même façon dans les différents habitats des sites. Leur distribution est liée à des habitats propres à chaque espèce.

(C) 2018 International Formulae Group. All rights reserved.

Mots clés : Avifaune, touracos, biogéographie, biodiversité, biologie de la conservation.

\section{Diversity, abundance and distribution of turacos (Musophagidae) in some natural environments in Central and Southeastern Côte d'Ivoire}

\begin{abstract}
The ecology and zoogeography of the turacos are little known in Côte d'Ivoire. Thus, in order to determine the diversity, abundance and distribution of the turacos, a study was carried out in the national parks of Banco, Azagny, in the in the Lamto Scientific Reserve and in the Yapo-Abbé classified forest, from August 2014 to July 2015. In the different environments, the inventory of the species of turacos belonging to the Musophagidae family was carried out using the point abundance index (PAI). In total, five species of turacos have been inventoried. They are: the yellow-billed turaco Tauraco macrorhynchus, the guinea turaco Tauraco persa, the great blue turaco Corythaeola cristata, the western plantain eater Crinifer piscator and the violet turaco Musophaga violacea. The results indicate that the Lamto Scientific Reserve has the greatest richness in
\end{abstract}


turaco and that Tauraco macrorhynchus is the only specie common to all the sites. On the order hand, Musophaga violacea is present only in the Lamto Scientific Reserve. The abundance of turacos varies from a site to another and habitat. In addition, turaco species are not distributed in the same way in the different habitats of the sites. Their distribution is linked to preferential habitats.

(C) 2018 International Formulae Group. All rights reserved.

Keywords: Avifauna, turacos, biogeography, biodiversity, biology of conservation.

\section{INTRODUCTION}

Le milieu naturel en Côte d'Ivoire s'est beaucoup dégradé au cours des dernières décennies. En effet, le couvert forestier qui était de 16 millions d'hectares avant 1960 a connu une transformation rapide pour se situer à moins de deux millions d'hectares de forêts naturelles (Koné et al., 2014). Les raisons de ce déclin sont principalement liées à l'agriculture extensive et à l'exploitation forestière non durablement gérée (Koné et al., 2014). La transformation des écosystèmes naturels entraîne leur fragmentation et la disparition des habitats et des niches écologiques des espèces animales (Franklin et al., 2002). Le superordre des oiseaux le mieux connu des vertébrés n'échappe pas à cette réalité (Lauginie, 2007). En effet, la principale menace qui pèse sur les oiseaux est la destruction de leurs habitats qui affecte leur survie. C'est le cas des touracos (Musophagidae) qui sont des espèces arboricoles vivant en forêts et dans les zones boisées (Borghesio et Ndang'ang'a, 2003).

Les Musophagidae sont parmi les oiseaux les plus caractéristiques de l'Afrique tropicale. En effet, ces oiseaux fréquentent divers habitats. Certaines espèces se rencontrent dans les forêts primaires denses, dans les milieux secondarisés, dans les milieux savanicoles et divers milieux ouverts alors que d'autres sont inféodées à des galeries forestières (Yaokokoré, 2001). La transformation des milieux naturels affecte donc leurs habitats. Les touracos jouent aussi un rôle important dans la régénération des écosystèmes forestiers à travers la dispersion des graines (Erard et Brosset, 2003). En outre, certaines espèces de touracos sont des spécialistes des forêts, caractéristiques de l'intérieur des forêts peu perturbées (Bennun et al., 2004). De ce fait, ces oiseaux pourraient être des bioindicateurs de la qualité des habitats forestiers.

Malgré leur importance écologique, les touracos ont fait l'objet de peu d'études spécifiques en Côte d'Ivoire où la plupart des études ornithologiques étaient axées sur la diversité générale (Yaokokoré-Beibro et al, 2010 ; Odoukpé et Yaokokoré-Beibro, 2014 ; Konan et Yaokokoré-Beibro, 2015). De ce fait, la parfaite connaissance des habitats préférentiels des espèces de touracos ainsi que leur distribution s'avèrent capitales ; ce, dans le cadre de la conservation de la biodiversité. La présente étude a été initiée dans ces milieux naturels qui constituent des refuges de la faune sauvage et supposés héberger un nombre élevé d'espèces de touracos au regard de la variabilité d'habitats qu'ils renferment. L'objectif de cette étude était donc de pallier l'insuffisance de données sur la famille des Musophagidae.

\section{MATERIEL ET METHODES Milieu d'étude}

Cette étude a été réalisée dans quatre milieux naturels en Côte d'Ivoire que sont les parcs nationaux du Banco (PNB) et d'Azagny (PNA), la Réserve Scientifique de Lamto (RSL) ainsi que la Forêt Classée de YapoAbbé (Figure 1).

Le Parc National du Banco (PNB) couvre une superficie de 3474 hectares et est situé en pleine agglomération d'Abidjan, capitale économique de la Côte d'Ivoire. Il s'étend entre $5^{\circ} 21^{\prime}$ et $5^{\circ} 25^{\prime}$ de latitude Nord et $4^{\circ} 01^{\prime}$ et $4^{\circ} 05^{\prime}$ de longitude Ouest (Lauginie, 2007). Le PNB est sous l'influence d'un climat de type subéquatorial à quatre 
saisons dont deux saisons des pluies et deux saisons sèches. Le PNB est une forêt dense sempervirente. Les arbres dominants dans les strates supérieures sont Turraeanthus africanus (Meliaceae) et Piptadeniastrum africanum (Fabaceae) (Lauginie, 2007). La faune du PNB est assez riche et diversifiée. Les groupes zoologiques les plus représentatifs sont les Mammifères, les Oiseaux et les Insectes (Lauginie, 2007). On y trouve 58 espèces de Mammifères (Bitty et al., 2013) et 166 espèces d'oiseaux dont la majorité est sédentaire (Kouadio, 2015).

Le Parc National d'Azagny (PNA) s'étend sur une superficie de 19400 hectares entre $5^{\circ} 9^{\prime} 5^{\circ} 17^{\prime}$ de latitude Nord et entre $4^{\circ} 47^{\prime}$ et $4^{\circ} 57^{\prime}$ 'de longitude Ouest. Il est situé au Sud de la Côte d'Ivoire à $130 \mathrm{~km}$ d'Abidjan dans le département de Grand-Lahou. Le climat du PNA est de type subéquatorial humide à quatre saisons (Lauginie, 2007). Le PNA est une mosaïque de formations végétales. On y trouve des forêts sempervirentes, des forêts secondaires, des jachères, des marécages, des savanes et des fourrés littoraux (Lauginie, 2007). La faune du PNA est constituée de singes, de buffles, d'éléphants, et de crocodiles avec une avifaune riche de 162 espèces (Lauginie, 2007).

La Forêt Classée de Yapo-Abbé (FCYA) couvre une superficie de 24592 hectares et s'étend entre $5^{\circ} 40^{\prime} 5^{\circ} 47^{\prime}$ de latitude Nord et entre $3^{\circ} 57^{\prime}$ et $4^{\circ} 11^{\prime}$ de longitude Ouest. Elle est située à $55 \mathrm{~km}$ d'Abidjan (SODEFOR, 1999). Le climat de la FCYA est de type subéquatorial à quatre saisons (SODEXAM, 2013). La FCYA est une forêt dense sempervirente dense caractérisée par l'abondance d'espèces végétales telles que Dacryodes klaineana (Burseraceae) Piptadeniastrum africanum (Fabaceae) et Heritiera utilis (Sterculiaceae). La faune de la FCYA est diversifiée. L'avifaune de cette forêt classée est riche de 225 espèces (Lauginie, 2007).

La Réserve Scientifique de Lamto (RSL) qui couvre une superficie de 2700 hectares, est située au centre de la Côte
d'Ivoire dans la sous-préfecture de Taabo entre $6^{\circ} 13^{\prime}$ de latitude Nord et $5^{\circ} 02^{\prime}$ de longitude Ouest. La RSL est sous influence d'un climat de type tropical humide à quatre saisons (Lauginie, 2007). La RSL est mosaïque forêts-savanes, constituée de galeries forestières, de forêt dense riveraine du fleuve Bandama, de forêts semi-décidues et de savanes préforestières à rôniers (Lauginie, 2007). La faune de la RSL se compose de mammifères, d'amphibiens et d'insectes qui y sont encore abondants, ainsi qu'une avifaune riche de 400 espèces (Lauginie, 2007).

\section{Matériel}

Pour la réalisation de cette étude, une paire de jumelles (10 x 50 BRESSER Hunter) a été utilisée pour observer les oiseaux. Un guide d'identification (Borrow et Demey, 2012) et la discographie de Chappuis (2000) ont servi à l'identification des oiseaux. Par ailleurs, un dictaphone (OLYMPUS VN-733 PC) a été utilisé pour l'enregistrement des vocalisations des oiseaux pour une identification ultérieure. Pour les prises de vues des oiseaux, un appareil photographique numérique (SONY DSC-HX 300 (X50)) a été utilisé et enfin un GPS (GPS MAP $60 \mathrm{C}_{\mathrm{X}}$ ) a servi à déterminer les coordonnées géographiques des différents points d'observation.

\section{Méthodes}

L'étude s'est déroulée d'août 2014 à juillet 2015. La méthode utilisée est celle des indices ponctuels d'abondance IPA (Bibby et al., 1992). Au total, quatre sites ont été visités (Figure 2) et au sein de chaque site, six habitats ont été retenus selon leurs caractéristiques écologiques.

Dans le PNB, les six habitats retenus sont les suivants. L'habitat1 est une mosaïque composée de forêt sur terre ferme, de forêt marécageuse et de jachères sur le cours inférieur de la rivière Banco. L'habitat 2 est une forêt sur terre ferme ayant subi un traitement sylvicole et dominée par Turraeanthus africanus (Meliaceae) avec un 
sous-bois peu dense. L'habitat 3 est un milieu anthropisé constitué d'habitations, de l'école forestière, de cultures vivrières et d'un arboretum. L'habitat 4 est une mosaïque de forêts composée de forêt sur terre ferme, de forêt ripicole et de forêt périodiquement inondée. L'habitat 5 est une portion de forêt sur terre ferme ayant subi des traitements sylvicoles intensifs avec bambouseraies et l'habitat 6 est constitué d'une portion de forêt sur terre ferme non perturbée.

Dans la FCYA, les six habitats échantillonnés sont les suivants : L'habitat 1 est constitué de plantations forestières âgées et de forêts naturelles aménagées avec un sousbois dense. L'habitat 2 est une forêt secondaire âgée parcourue de pistes de débardage. L'habitat 3 est une forêt secondaire âgée renfermant une enclave d'exploitations agricoles de papayers, bananiers, colatiers et une ancienne cacaoyère. L'habitat 4 est constitué de plantations forestières avec des bas-fonds formés de bambouseraies. L'habitat 5 est une forêt secondaire âgée dominée par de grands arbres à canopée fermée et l'habitat 6 est une forêt secondaire âgée avec des pistes de planting d'arbres.

Dans le PNA, les six habitats retenus présentent les caractéristiques suivantes: l'habitat 1 est une forêt dense sempervirente à canopée fermée; l'habitat 2 est constitué d'une forêt secondaire âgée et d'une zone marécageuse à Raphia; l'habitat 3 est un habitat constitué de forêts secondaires dégradées et de jachères; l'habitat 4 est constitué de fourrés parsemés de Raphias et de palmiers à huile; l'habitat 5 est une savane avec un tapis herbacé touffu parsemée de rôniers et l'habitat 6 est une savane parsemée de palmiers à huile et de quelques arbustes.

Dans la RSL, les six habitats échantillonnés sont les suivants : l'habitat 1 est une savane préforestière à rôniers ; l'habitat 2 est une forêt semi-décidue; l'habitat 3 est une savane à rônier avec une relique de forêt galerie; l'habitat 4 est une lisière entre une forêt galerie et la savane préforestière; l'habitat 5 est une galerie forestière claire à canopée plus ou moins fermée et l'habitat 6 est une forêt dense riveraine du fleuve Bandama avec de grands arbres.

Dans chaque habitat, sept points d'écoute ont été installés le long des pistes d'aménagement. La distance comprise entre deux points d'écoute consécutifs a été de 300 $\mathrm{m}$. Les inventaires sont faits de façon journalière, de $6 \mathrm{~h}$ à $12 \mathrm{~h}$ puis de $14 \mathrm{~h} 30$ à 18 h. Le temps mis dans chaque point d'écoute est de 20 minutes au cours duquel les espèces de touracos et leur abondance ont été notées. Chaque site a été visité une fois chaque deux mois et la durée d'une visite de site est de six jours. Les données recueillies ont permis de déterminer différents paramètres que sont la richesse spécifique (S) qui exprime le nombre d'espèces observées dans un peuplement $\left(\mathrm{S}=\sum\right.$ espèces ) ; la fréquence relative $(\mathrm{Fr})$ qui est l'importance relative de chacune des espèces par rapport à toutes celles enregistrées dans un habitat donné $\left(\mathrm{F}_{\mathrm{r}}=(\mathrm{ni} / \mathrm{N})\right.$ x100 où ni $=$ effectif de la population d'espèce $\mathrm{i}$ et $\mathrm{N}=$ somme des effectifs des espèces constituant le peuplement); l'indice de diversité de Shannon (H') qui permet de calculer le niveau de diversité du milieu $\left(\mathrm{H}^{\prime}=-\Sigma(\mathrm{ni} / \mathrm{N}) \ln (\mathrm{ni} / \mathrm{N})\right)$ ainsi que l'indice d'équitabilité de Piélou (J) qui permet de comparer la diversité de deux peuplements ayant des richesses spécifiques différentes ( $\mathbf{J}=\mathrm{H}^{\prime}$ / H'max avec H'max $=\ln$ S).

Ces données ont également permis de
faire une analyse factorielle de
correspondance (AFC) pour montrer la
distribution des espèces de touracos en
fonction des différents sites.




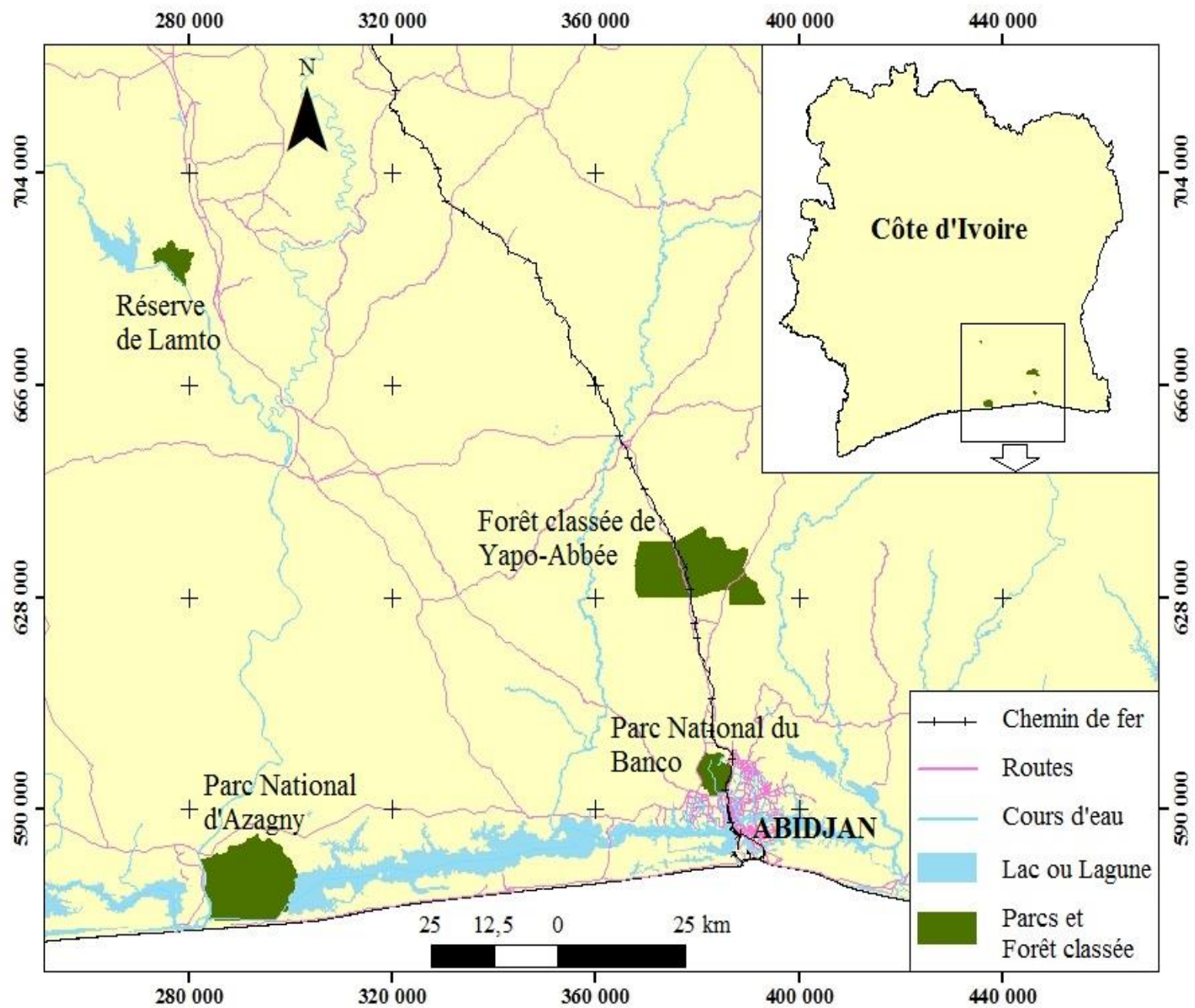

Figure 1 : Localisation des sites d'étude des touracos en Côte d'Ivoire.

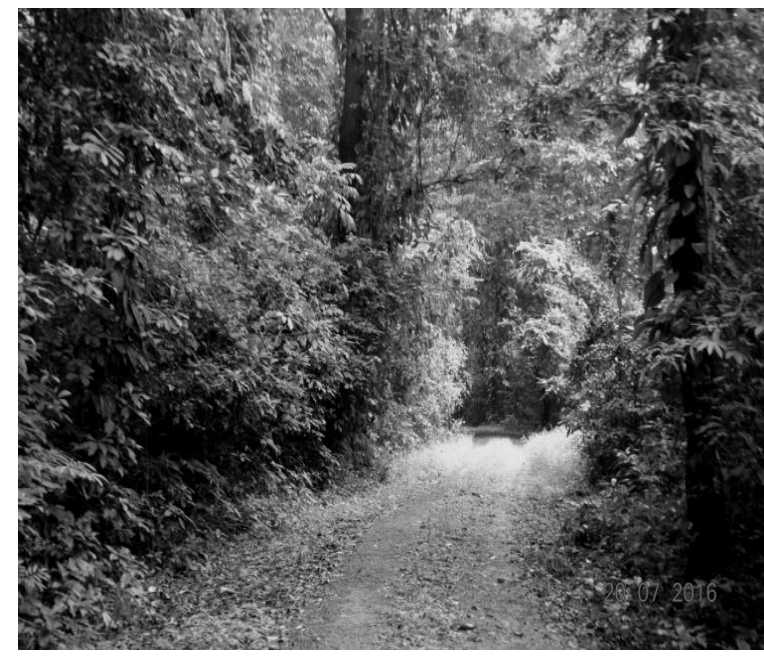

a. PNB. $\left(5^{\circ} 23^{\prime} 41.1^{\prime \prime} \mathrm{N} / 4^{\circ} 03^{\prime} 25.8^{\prime \prime} \mathrm{O}\right)$

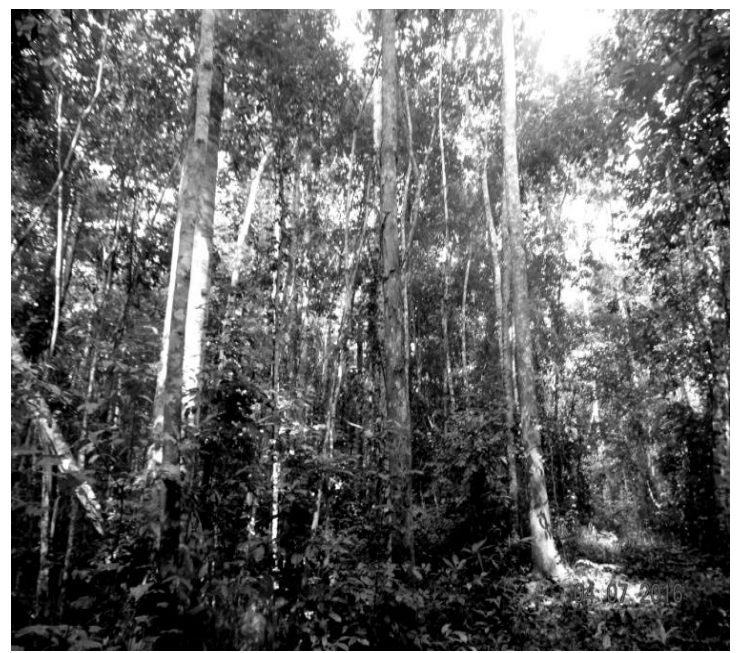

b. FCYA. $\left(5^{\circ} 42^{\prime} 36.9^{\prime \prime} \mathrm{N} / 4^{\circ} 04^{\prime} 38.3^{\prime \prime} \mathrm{O}\right)$ 


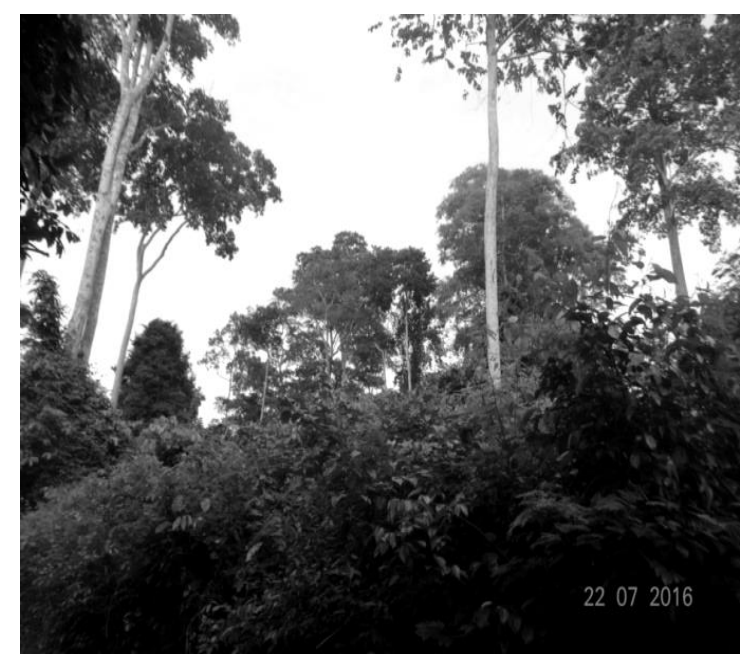

c. PNA. $\left(5^{\circ} 14^{\prime} 26.7^{\prime \prime} \mathrm{N} / 4^{\circ} 48^{\prime} 49.9^{\prime \prime} \mathrm{O}\right)$

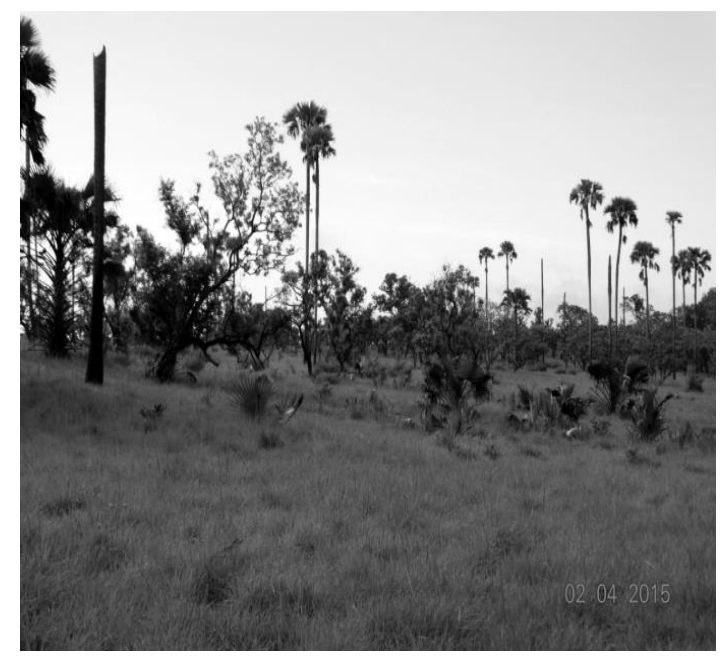

d. RSL. $\left(6^{\circ} 13^{\prime} 30.7^{\prime \prime} \mathrm{N} / 5^{\circ} 01{ }^{\prime} 13.3^{\prime \prime} \mathrm{O}\right)$

Figure 2: Quelques faciès de la végétation des sites visités.

PNB : Parc national du Banco PNA : Parc national d'Azagny $\quad$ FCYA : Forêt classée de Yapo-Abbé

RSL : Réserve scientifique de Lamto.

\section{RESULTATS}

Richesse spécifique et abondance globales dans l'ensemble des sites

L'étude réalisée dans les quatre sites a permis d'inventorier cinq espèces de touracos. Il s'agit du touraco à gros bec Tauraco macrorhynchus (Fraser, 1839) (Figure 3), du touraco vert Tauraco persa (Linné, 1758) (Figure 4), du touraco géant Corythaeola cristata (Vieillot, 1816) (Figure 5), du touraco violet Musophaga violacea Isert, 1788 (Figure

6) et du touraco gris Crinifer piscator (Boddaert, 1783) (Figure 7). Dans l'ensemble des sites, 253 individus de Tauraco macrorhynchus ont été enregistrés. L'abondance relative globale de Tauraco persa est de 111 individus. Seuls 20 individus de Corythaeola cristata ont été recensés. L'abondance globale de Musophaga violacea est de 34 individus alors que celle de Crinifer piscator est de 68 individus. L'abondance relative globale est donc de 486 individus avec un effectif cumulé de 919 individus.

\section{Diversité et abondance des espèces de} touracos dans chaque site

Les résultats obtenus montrent que la composition spécifique des touracos et les indices de diversité varient d'un site à l'autre (Tableau 1).

Dans le PNB, une seule espèce de touraco a été inventoriée. Il s'agit du Tauraco macrorhynchus avec une abondance relative de 112 individus. Le PNB est le site le moins diversifié.

Au sein de la FCYA, deux espèces de touracos ont été inventoriées. Ce sont: le touraco à gros bec Tauraco macrorhynchus et le touraco géant. Le touraco à gros bec Tauraco macrorhynchus est l'espèce la plus dominante. Son abondance relative est de 90 individus, ce qui représente une fréquence relative de 97,83\%. Par ailleurs, Corythaeola cristata est l'espèce la moins abondante avec une abondance relative de deux individus soit une fréquence relative de $2,17 \%$. La FCYA est le site le moins diversifié $\left(\mathrm{H}^{\prime}=0,10 ; \mathrm{J}=\right.$ $0,06)$. 
Dans le PNA, quatre espèces de touracos ont été inventoriées; il s'agit de Tauraco macrorhynchus, Tauraco persa, Corythaeola cristata et de Crinifer piscator. Tauraco macrorhynchus, est l'espèce dominante avec une abondance relative de 33 individus, ce qui représente une fréquence relative de $35,11 \%$ alors que Corythaeola cristata est l'espèce la moins abondante avec une abondance relative de six individus, soit une fréquence relative de $6,38 \%$. Le PNA est un site diversifié $\left(\mathrm{H}^{\prime}=1,26 ; \mathrm{J}=0,78\right)$.

Dans la RSL, toutes les cinq espèces de touracos ont été inventoriées. Tauraco persa est l'espèce dominante, avec une abondance relative de 85 individus, soit une fréquence relative de $45,25 \%$ alors que Corythaeola cristata est l'espèce la moins abondante avec une abondance de 12 individus soit une fréquence relative de $6,38 \%$. La RSL est le site le plus diversifié $\left(\mathrm{H}^{\prime}=1,40 ; \mathrm{J}=0,87\right)$. $\mathrm{La}$ composition spécifique des touracos est élevée dans la RSL alors qu'elle est faible dans le PNB.

\section{Distribution des espèces de touracos par site et par habitat}

Les résultats obtenus montrent que la distribution des espèces de touracos varie d'un site à l'autre et d'un habitat à un autre.

Dans le PNB, Tauraco macrorhynchus se distribue dans tous les habitats constitués essentiellement de forêts secondaires âgées. Cependant, l'espèce est absente en milieu anthropisé constitué de cultures vivrières, d'habitations et d'arboretum.

$\mathrm{Au}$ sein de la FCYA, Tauraco macrorhynchus est présent dans tous les habitats constitués de plantations forestières, de forêts naturelles aménagées et de forêts secondaires âgées. Par ailleurs, Corythaeola cristata a été observé en forêts secondaires âgées.

Dans le PNA, Tauraco
macrorhynchus, Tauraco persa et
Corythaeola cristata se distribuent dans les
formations forestières alors Crinifer piscator

se rencontre dans les savanes côtières et les fourrés littoraux. En outre, Tauraco macrorhynchus et Corythaeola cristata fréquentent les forêts primaires et les forêts secondaires âgées tandis que Tauraco persa se distribue essentiellement dans les forêts secondaires et dans les jachères.

Dans la RSL, Tauraco macrorhynchus se distribue principalement dans la forêt dense riveraine du fleuve Bandama. De même, Corythaeola cristata se rencontre dans la forêt dense riveraine du fleuve Bandama et parfois dans les galeries forestières. Tauraco persa se distribue essentiellement dans les galeries forestières et dans les forêts semi-décidues alors que Musophaga violacea est présent dans la forêt riveraine et surtout dans les galeries forestières. Par ailleurs, Crinifer piscator se rencontre majoritairement dans la savane préforestière à rôniers mais se retrouve quelquefois dans les galeries forestières (Tableau 2).

Toutefois, l'analyse factorielle des correspondances (AFC) appliquée aux variables espèces et sites, fait ressortir deux groupes écologiques sur la base du premier axe factoriel qui présente la plus grande inertie $(92,42 \%)$. Le premier groupe, constitué de quatre espèces de touracos, est corrélé positivement à cet axe. Cependant, l'observation du deuxième axe factoriel exprimant une inertie de $7,56 \%$, montre une nette ségrégation des espèces de ce premier groupe. En effet, dans la partie positive du deuxième axe, deux espèces de touracos, Tauraco persa et Musophaga violacea sont associés à la RSL. Par contre, dans sa partie négative, deux autres espèces de touracos, Crinifer piscator et Corythaeola cristata sont associés au PNA. Cependant, Crinifer piscator est beaucoup plus associé à la RSL qu'au PNA. Le second groupe, composé d'une seule espèce de touraco, est corrélé négativement au premier axe factoriel. Il s'agit de Tauraco macrorhynchus qui est fortement associé au PNB et à la FCYA (Figure 8). 


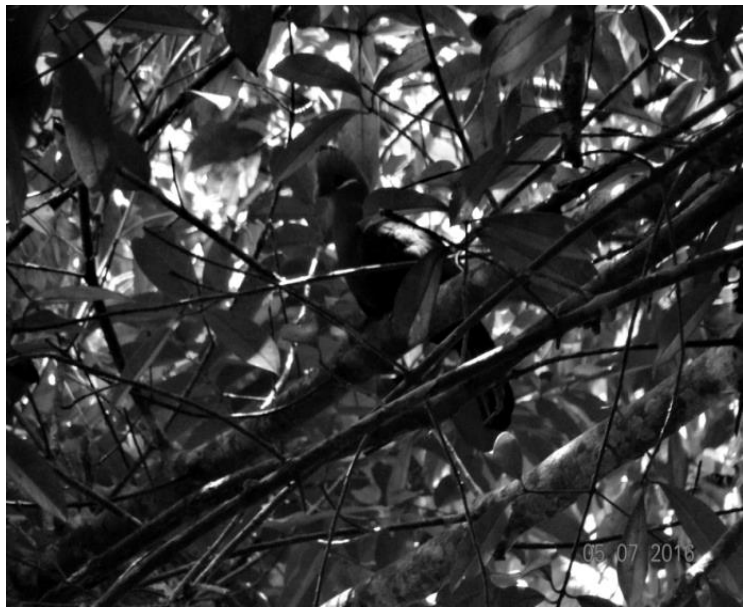

Figure 3 : Touraco à gros bec Tauraco macrorhynchus (Fraser, 1839).

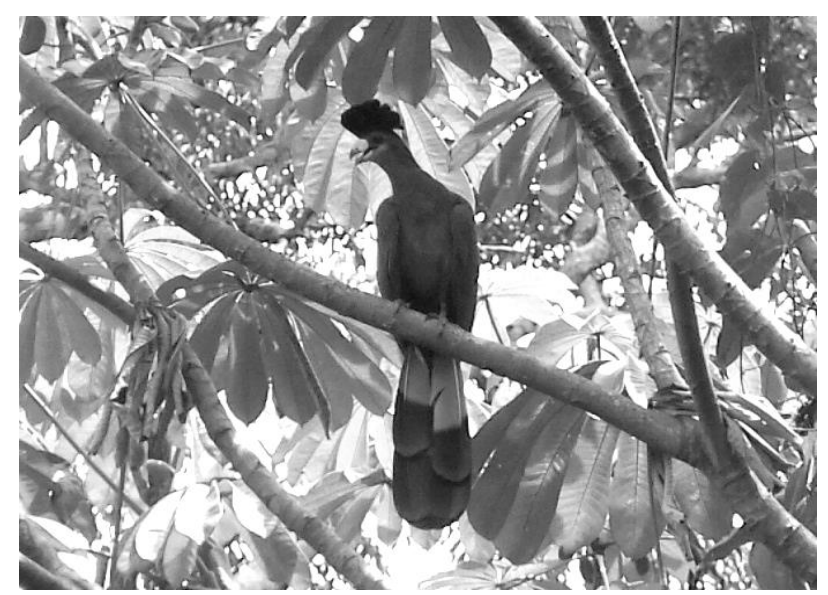

Figure 5 : Touraco géant Corythaeola cristata (Vieillot, 1816).

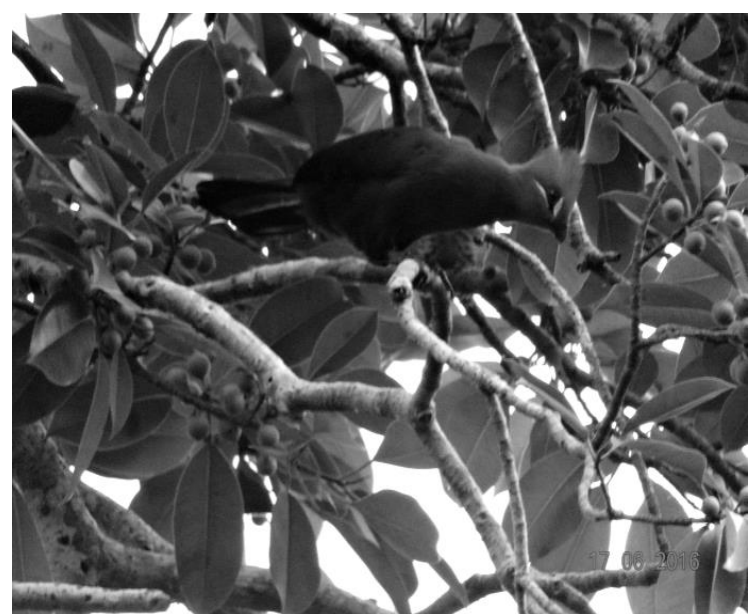

Figure 4 : Touraco vert Tauraco persa (Linné. 1758).

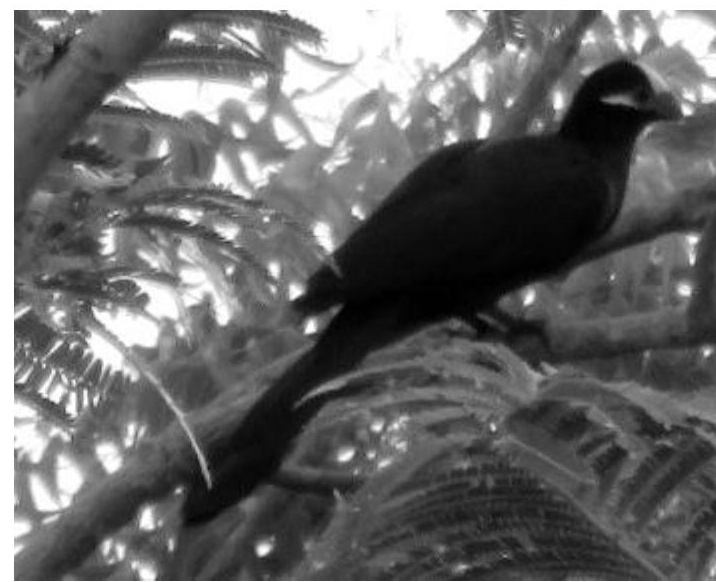

Figure 6 : Touraco violet Musophaga violacea (Isert, 1788).

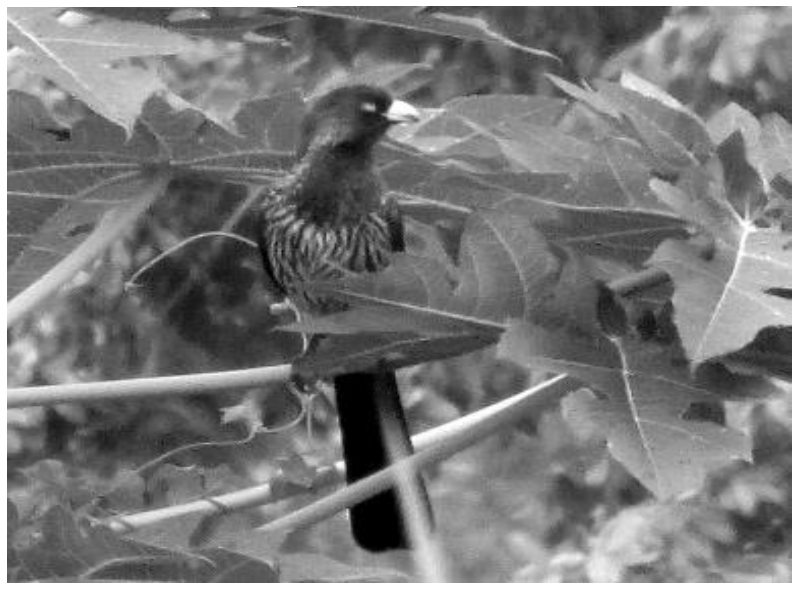

Figure 7: Touraco gris Crinifer piscator (Boddaert, 1783). 
O. M. OKON et al. / Int. J. Biol. Chem. Sci. 12(4): 1851-1864, 2018

Tableau 1: Indice ponctuel d'abondance et Fréquence relative des différentes espèces de touracos selon les sites prospectés.

\begin{tabular}{|c|c|c|c|c|c|c|c|c|}
\hline \multirow[t]{2}{*}{ Espèces } & \multicolumn{2}{|c|}{ PNB } & \multicolumn{2}{|c|}{ FCYA } & \multicolumn{2}{|c|}{ PNA } & \multicolumn{2}{|c|}{ RSL } \\
\hline & IPA & $\operatorname{Fr}(\because \%)$ & IPA & $\operatorname{Fr}(\cdot \%)$ & IPA & $\operatorname{Fr}(\because \%)$ & IPA & $\operatorname{Fr}(\cdot \%)$ \\
\hline Tauraco persa & - & - & - & - & 26 & 27,66 & 85 & 45,21 \\
\hline Tauraco macrorhynchus & 112 & 100 & 90 & 97,83 & 33 & 35,11 & 18 & 9,57 \\
\hline Crinifer piscator & - & - & - & - & 29 & 30,85 & 39 & 20,74 \\
\hline Musophaga violacea & - & - & - & - & - & - & 34 & 18,09 \\
\hline Corythaeola cristata & - & - & 2 & 2,17 & 6 & 6,38 & 12 & 6,38 \\
\hline TOTAL & 112 & 100 & 92 & 100 & 94 & 100 & 188 & 100 \\
\hline $\mathbf{S}$ & \multicolumn{2}{|c|}{1} & \multicolumn{2}{|c|}{2} & \multicolumn{2}{|c|}{4} & \multicolumn{2}{|c|}{5} \\
\hline H' & \multicolumn{2}{|c|}{-} & \multicolumn{2}{|c|}{0,10} & \multicolumn{2}{|c|}{1,26} & \multicolumn{2}{|c|}{1,40} \\
\hline $\mathbf{J}$ & \multicolumn{2}{|c|}{-} & \multicolumn{2}{|c|}{0,06} & \multicolumn{2}{|c|}{0,78} & \multicolumn{2}{|c|}{0,87} \\
\hline
\end{tabular}


O. M. OKON et al. / Int. J. Biol. Chem. Sci. 12(4): 1851-1864, 2018

Tableau 2 : Abondance des espèces de touracos selon les habitats des sites échantillonnés.

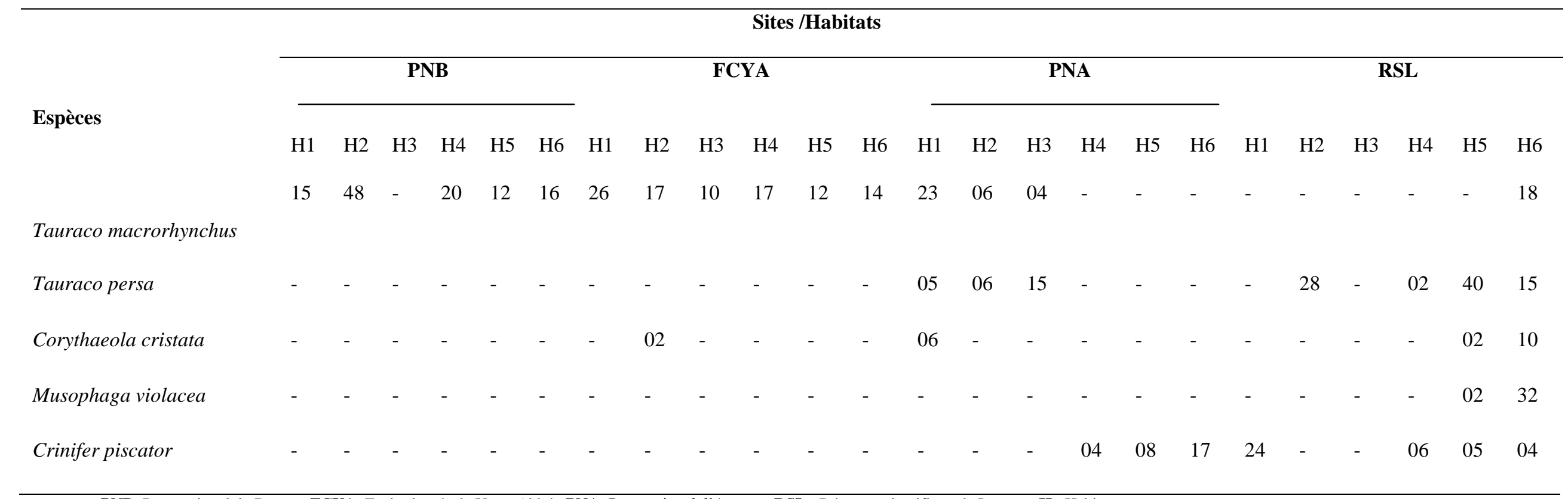

PNB: Parc national du Banco ; FCYA: Forêt classée de Yapo-Abbé ; PNA: Parc national d’Azagny; RSL : Réserve scientifique de Lamto ; H : Habitat. 


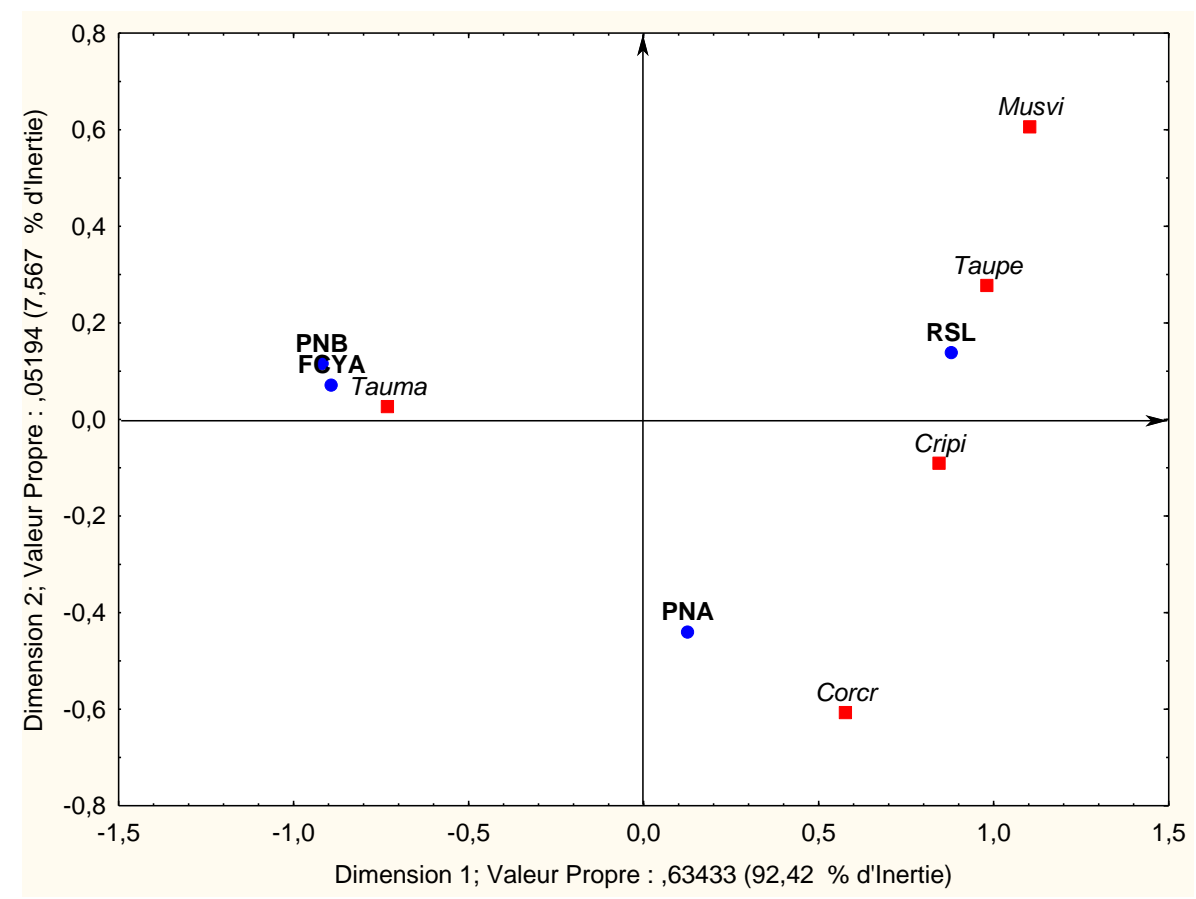

Figure 8 : Distribution des espèces de touracos en fonction des différents sites d'étude. Taupe: Tauraco persa; Tauma: Tauraco macrorhynchus; Cripi: Crinifer piscator Corcr: Corythaeola cristata; Musvi: Musophaga violacea PNB : Parc national du Banco ; FCYA : Forêt classée de Yapo-Abbé PNA : Parc national d'Azagny ; RSL : Réserve Scientifique de Lamto.

\section{DISCUSSION}

Au total, cinq espèces de touracos ont été inventoriées dans l'ensemble des sites visités. Ce sont les cinq espèces de touracos dont la présence a été signalée en Côte d'Ivoire (Borrow et Demey, 2012).

Des quatre sites échantillonnés, la RSL est le site le plus riche en espèces de touracos. Par contre, le PNB en compte moins. En effet, la RSL renferme des habitats variés tels que la savane préforestière, des galeries forestières, des forêts semi-décidues et la forêt dense riveraine du fleuve Bandama (Lauginie, 2007). Le PNB est moins riche en espèces de touracos car il renferme globalement un seul type formation végétale. Ce site est constitué essentiellement de forêts denses sempervirentes (Lauginie, 2007). Il ressort de l'analyse de ces résultats que les sites qui renferment des habitats variés ont une richesse spécifique en touracos élevée. La richesse spécifique en touracos d'un milieu serait corrélée à la variété d'habitats qu'il renferme et à leurs caractéristiques écologiques. Les résultats obtenus montrent aussi une disparité dans la distribution des espèces de touracos dans les différents habitats des sites visités. Chaque espèce de touraco est inféodée à un habitat préférentiel. La distribution des espèces de touracos dans les milieux naturels est liée à des habitats préférentiels car, selon Yeboah et al. (2008), dans des travaux réalisés dans le Parc National de Kakum, dans la Région Centrale du Ghana, les habitats préférentiels contribuent à la distribution des touracos dans leurs milieux. En effet, Tauraco macrorhynchus préfère les forêts primaires alors que Tauraco persa occupe préférentiellement les formations arborées secondaires. Ce choix préférentiel d'habitats selon Erard et Brosset (2003), leur permet d'éviter les compétitions interspécifiques car 
ces deux espèces très voisines s'exclurent territorialement. Tauraco macrorhynchus est donc une espèce spécialiste des forêts comme l'a également mentionné Sekercioglu (2002). Par ailleurs, Corythaeola cristata se distribue dans les forêts denses primaires, les forêts secondaires, les galeries forestières ainsi que les îlots forestiers. L'adaptation de l'espèce à ces différents habitats est due probablement au fait qu'elle a une grande amplitude écologique, ce qui lui permet de vivre dans des habitats forestiers variés car selon Sekercioglu (2002), le touraco géant Corythaeola cristata est une espèce généraliste des forêts. Cependant, l'espèce est absente dans le PNB. Cette absence s'expliquerait par l'enclavement du PNB et à la pression anthropique qui s'exerce sur ce parc, avec la disparition des couloirs écologiques (Kouadio, 2015). En outre, l'espèce pourrait aussi être victime du braconnage.

Crinifer piscator se distribue dans les savanes côtières, les fourrés littoraux, la savane préforestière à rôniers et les galeries forestières. L'espèce serait donc inféodée aux savanes et aux milieux ouverts. Ainsi, l'absence de cette espèce dans le PNB et la FCYA serait liée à l'absence de formations savanicoles et de milieux ouverts de grande étendue au sein de ces sites. Cette préférence de Crinifer piscator pour les milieux ouverts et dégradés a également été mis en exergue dans les milieux forestiers de substitution (plantation et jachères) du Bénin (Lougbegnon et al., 2010).

Par ailleurs, Musophaga violacea est présent uniquement dans la RSL au sein de laquelle il se distribue dans la forêt riveraine $\mathrm{du}$ fleuve Bandama, dans les galeries forestières et les îlots forestiers. En effet, de tous les sites visités, la RSL est le seul site situé en zone de savane. Musophaga violacea ne se distribue pas partout en Côte d'Ivoire. L'espèce serait la seule espèce de touraco typiquement de savane.

\section{Conclusion}

L'étude réalisée dans ces différents sites avait pour objectif de contribuer à une meilleure connaissance de l'écologie et de la distribution des touracos en Côte d'Ivoire. Les résultats obtenus ont confirmé la présence des cinq espèces de touracos en Côte d'Ivoire. La richesse spécifique varie d'un site à l'autre. Par ailleurs, la RSL est le site le plus riche en espèces de touracos, avec cinq espèces alors que le PNB en compte moins, avec une seule. En outre, l'étude a montré que les espèces de touracos ne sont pas distribuées de la même façon dans les habitats des différents sites. Leur distribution est fonction des types d'habitats préférentiels à chaque espèce. Cependant des études complémentaires relatives aux ressources alimentaires et à l'effet des variations saisonnières devraient être menées afin de permettre une meilleure compréhension de la distribution des touracos dans leurs milieux

\section{CONFLIT D'INTERETS}

Les auteurs du présent manuscrit déclarent qu'il n'existe aucun conflit d'intérêt relatif à celui-ci.

\section{CONTRIBUTIONS DES AUTEURS}

OMO : Récoltes des données de terrain, rédaction du manuscrit; EMK : Récolte des données de terrain, aide à la réalisation des tests statistiques et correction du manuscrit; KPK : Analyse des données et correction du manuscrit; HKY-B : Garant scientifique, conception $\mathrm{du}$ thème de recherche et supervision scientifique de l'étude.

\section{REMERCIEMENTS}

Nous remercions la Direction Générale de l'OIPR (Office Ivoirien des Parcs et Réserves) ainsi que la Direction Générale de la SODEFOR (Société de Développement des Forêts) de nous avoir accordé les autorisations d'accès dans les pars nationaux du Banco et 
d'Azagny, de la Reserve Scientifique de Lamto et la Forêt classée de Yapo-Abbé. Sans ces différentes autorisations, cette étude n'aurait pas été possible. Nos sincères remerciements vont à l'endroit des docteurs ODOUKPE Kadio Saint-Guillaume et KOUE bi Tih Mathieu pour leurs critiques et suggestions dans l'orientation de la rédaction $\mathrm{du}$ manuscrit. Nous exprimons notre reconnaissance aux agents de l'OIPR et de la SODEFOR pour leur assistance technique durant cette étude. Enfin, nous remercions Mrs Yaoba et Kouamé Julien, nos guides pour leur aide pendant les inventaires.

\section{REFERENCES}

Bennun L, Davies G, Howell K, Newing H, Lankie M. 2004. La biodiversité des forêts d'Afrique : Manuel pratique de recensement des vertébrés. UK, 1st edn Earthwatch Institute (Europe). P.180.

Bibby C, Burgess N, Hill D. 1992. Birds Census Techniques. Academic Press: London; 134.

Bitty EA, Kadjo B, Gonedele BS, Okon OM, Kouassi KP. 2013. Inventaire de la faune mammalogique d'une forêt urbaine, Parc National du Banco, Côte d'Ivoire. Int. J. Biol. Chem. Sci., 7(4): 1678-1687. DOI: http: //dx.doi.org/10.4314/ijbcs.v7i4.23

Borghesio L, Ndang'ang'a KP. 2003. Habitat selection and the conservation status of Fischer's turaco Tauraco fischeri on Unguja, Tanzania. Oryx, 37(4): 444-453. DOI:

http://doi.org/10.1017/S0030605303000 814

Borrow N, Demey R. 2012. Guide des Oiseaux de l'Afrique de l'Ouest. Delachaux et Nestlé : Paris.

Chappuis C. 2000. African Bird Sounds. Society ornithology: France, Paris, 15 CD.

Deniveau JL. 1976. Principales caractéristiques physionomiques et floristiques des formations forestières de
Lamto (Moyenne Côte d'Ivoire). Annale de l'Université d'Abidjan, E, 9: 26-33.

Erard C, Brosset A. 2003. Les oiseaux de la région du Nimba : Structure et fonctionnement des peuplements, in le peuplement animal du mont nimba (Guinée, Côte d'Ivoire, Liberia). Mémoires $d u$ Muséum National d'Histoire Naturelle, 190 : 639-685.

Franklin JF, Spies TA, Van Pelt R, Carey AB, Thornburgh DA, Berg DR, Lindenmayer DB, Harmon ME, Keeton WS, Shaw DC. 2002. Disturbances and structural development of natural forest ecosystems with silvicultural implications, using Dauglas-fir forests as an example. Forest Ecology and Management, 155: 399-423. DOI: https://doi.org/10.1016/S0378-1127 (01) 00575.8

Konan EM, Yaokokoré-Beibro KH. 2015. Variation du peuplement aviaire des écosystèmes lacustres de la ville de Yamoussoukro, centre de la Côte d'Ivoire. Int. J. Biol. Chem. Sci., 9(6) : 2566-2581.

DOI : http://dx.doi.org/10.4314/ijbcs.v9i6.5

Koné M, Kouadio YL, Neuba DFR, Malan DF, Coulibaly L. 2014. Evolution de la couverture forestière de la Côte d'Ivoire des années 1960 au début du 21e siècle. International Journal of Innovation and Applied Studies, 7(2): 782-794. http://www.ijias.Issr-journal.org

Kouadio KP. 2015. Diversité avifaunique, écologie des Pycnonotidae et des Turdidae du Parc national du Banco (Côte d'Ivoire). Thèse de Doctorat, Université Félix Houphouët Boigny de Cocody, p.240.

Lauginie F. 2007. Conservation de la nature et des aires protégées en Côte d'Ivoire. CEDA/NEI, Abidjan.

Lougbegnon TO, Codjia JC, Libois RM. 2010. Distribution de l'avifaune des milieux forestiers de substitution 
(plantation et jachères) au sud du Bénin en relation avec les facteurs de l'habitat. Int. J. Biol. Chem. Sci., 4(4) : 1191-1216. DOI:

http://dx.doi.org/10.4314/ijbcs.v4i4.6305 6

Odoukpé KSG, Yaokokoré-Beibro KH. 2014. Avifaune des champs de riz de la zone humide de Grand-Bassam (Côte d'Ivoire). Int. J. Biol. Chem. Sci., 8(4): 1458-1480.

DOI: http://dx.doi.org/10.4314/ijbcs.v8i4.10

Sekercioglu CH. 2002. Effects of forestry practices on vegetation structure and bird community of Kibale National Park, Uganda. Biological Conservation, 107: 229-240.

DOI:

https://doi.org/10.1016/S0006-3207 (02)00097.6

SODEXAM. 2013. Données météorologiques de la Région de l'Agnéby-Tiassa.

SODEFOR. 1999. Plan d'aménagement de la forêt classée de Yapo-Abbé. 68P.

Yaokokoré-Beibro KH. 2001. Avifaune des forêts classées de l'Est de la Côte d'Ivoire: Données sur l'écologie des espèces et effet de la déforestation sur les peuplements cas des forêts classées de la Béki et de la Bossématié (Abengourou). Thèse de Doctorat, Université de Cocody, p.245.

Yaokokoré-Beibro KH, N'guessan AM, Odoukpé KSG, Zouzou EJ, N'douba V, Kouassi KP. 2010. Premières données sur les oiseaux de la zone humide d'importance internationale de GrandBassam (Côte d'Ivoire). Int. J. Biol. Chem. Sci., 4(6): 2169-2180. DOI: http://dx.doi.org/10.4314/ijbcs.v4i6.6497 0

Yeboah S, Deikumah JP, Henaku-Owusu EE. 2008. The status of three species of turacos in the Kakum Conservation Area in the Central Region, Ghana. West African journal of Applied Ecology, 13: 104-110. DOI: 10.4314/wajae.v13i1.40578. 